\title{
Significados sobre la prevención del riesgo de VIH en cibernautas con trastorno bipolar
}

\author{
Dulce María Galarza Tejada, ${ }^{1}$ Ramiro Caballero Hoyos, ${ }^{2}$ Luciana Ramos Lira ${ }^{3}$
}

Artículo original

\section{SUMMARY}

Introduction

The persons with affective bipolar disorder (ABD) constitute a vulnerable group to the infection of HIV; in spite of that, there has been little investigation about the preventive culture in this population.

\section{Objective}

To identify the meanings and practices about the prevention of the risk of HIV infection with persons having bipolar disorders, active members of the weblog "Foro Bipolarmexico".

\section{Methodology}

A quality ethnographic type study was carried out on the Mexican weblog, directed by and for persons with ABD. The sampling of forums of expression published from 2007 to 2010 were positive. An analysis of the contained inductive thematic was conducted and the ATLAS.ti was utilized for the process of the information.

\section{Results}

The meanings and the conditions implied in the construction of the practices on the prevention of the risk of transmission of HIV cover three levels: a) physic-biologic, where the symptomatology associated with the disorder is identified, and the use of medications are implicated in the altercation of desires and sexual practices; before this, the bloggers propose the execution of activities which would permit the canalization of sexual impulse, the change of medication and use of preservatives; b) the micro-social, in which enhanced conditions like communication deficiency with their psychiatrists and similarities on the disorders and their effects on sexuality, for which they promote open discussion on the changes experienced; and c) the macro-social, where the stigma of the disease is expressed in solitude and the difficulty of stable couples to relate, conditions that could counteract, so they suggested, with the social recognition of their potentials.

The emphasis on the recuperation of significance constructed socially clearly showed that it is urgent to consider directed towards not only preventive interventions, but also to micro and macro social conditions which have a part in the vulnerability and infection of HIV.

Key words: Bipolar disorder, prevention \& control, HIV, weblog, social support networks.

\section{RESUMEN}

Introducción

Las personas con trastorno afectivo bipolar (TAB) constituyen un grupo vulnerable al contagio del $\mathrm{VIH}$; a pesar de ello, poco se ha investigado sobre la cultura preventiva en esta población.

\section{Objetivo}

Identificar los significados y prácticas sobre la prevención del riesgo de transmisión de $\mathrm{VIH}$ en personas con trastorno bipolar, miembros activos del weblog "Foro Bipolarmexico".

\section{Metodología}

Se realizó un estudio cualitativo de tipo etnográfico en una weblog mexicana, dirigida por y para personas con TAB. El muestreo de foros de expresión publicados de 2007 a 2010 fue propositivo. Se realizó un análisis de contenido temático inductivo y para el procesamiento de la información se utilizó el software ATLAS.ti.

\section{Resultados}

Los significados y las condiciones implicadas en la construcción de prácticas sobre la prevención del riesgo de transmisión de $\mathrm{VIH}$ abarcan tres niveles: a) el psicobiológico, donde se identifica que la sintomatología asociada al trastorno y el uso de medicamentos están implicados en la alteración de deseos y prácticas sexuales; ante esto los blogueros proponen realizar actividades que permitan la canalización del impulso sexual, el cambio de medicamentos y utilizar preservativos; b) el microsocial, en el cual destacaron condiciones como la deficiente comunicación con sus psiquiatras y parejas sobre el trastorno y su efecto en la sexualidad, por lo que ellos promueven la discusión abierta sobre los cambios experimentados y c) el macrosocial, donde el estigma de la enfermedad se expresa en el aislamiento y dificultad para relacionarse con parejas estables, condiciones que podrían contrarrestarse, según sugieren, con el reconocimiento social de sus potencialidades.

El énfasis en la recuperación de significados construidos socialmente evidenció que es apremiante considerar intervenciones preventivas dirigidas tanto a las prácticas de riesgo individuales como hacia las condiciones microsociales y macrosociales que participan en la vulnerabilidad al contagio de $\mathrm{VIH}$.

Palabras clave: Trastorno bipolar, prevención, VIH, weblog, redes de apoyo social.

- Facultad de Psicología, Universidad Autónoma de San Luis Potosí, S.L.P. México.

2 Unidad de Investigación en Epidemiología Clínica, Instituto Mexicano del Seguro Social, Delegación Colima. Col. México.

Dirección de Investigaciones Epidemiológicas y Psicosociales. Instituto Nacional de Psiquiatría Ramón de la Fuente Muñíz.

Correspondencia: Dulce María Galarza Tejada. Facultad de Psicología, Universidad Autónoma de San Luis Potosí. Carretera Central Km. 424.5, 78494, San Luis Potosí, SLP, México. Tel: (444) 822 - 2215, Tel. particular: (444) 243 - 5810. E-mail: galarza_dulce@yahoo.com.mx

Recibido: 7 de marzo de 2012. Aceptado: 14 de octubre de 2012. 


\section{INTRODUCCIÓN}

Las personas con trastornos mentales graves (TMG), como el trastorno afectivo bipolar (TAB), constituyen un grupo de alta vulnerabilidad para el riesgo de infección por el virus de inmunodeficiencia humana (VIH). Joska et al. ${ }^{1}$ reportaron para esta población una seroprevalencia elevada de $\mathrm{VIH}$ (10\%, rango 0-59.3\%), con una tendencia creciente en el tiempo. Antes de 1996, la seroprevalencia variaba entre 0 y $22.9 \%$, mientras que después de 1996 fluctuaba entre 6 y 59.3\%.

Los motivos por los que las personas con TAB son comparativamente más vulnerables a esta infección, considerando a personas con otros trastornos son: 1. tienen mayor frecuencia de prácticas sexuales que los pacientes esquizoafectivos ${ }^{2}$ y los depresivos; ; ${ }^{3,4}$ 2. en las mujeres existe un número más alto de infecciones de transmisión sexual (ITS) en el curso de su vida que en los pacientes depresivos unipolares; ${ }^{5}$ 3. tienen mayor seropositividad; ${ }^{6}$ y 4 . presentan más factores de riesgo. ${ }^{7}$

Un estudio realizado en Estados Unidos en población con TAB encontró que el mayor uso de sustancias psicoactivas, la menor gravedad psiquiátrica y la presencia de un episodio maniaco reciente eran factores predictores significativos para el riesgo de transmisión de VIH. ${ }^{8}$ Por su parte, un estudio realizado en adolescentes nigerianos con bipolaridad encontró que la comorbilidad, principalmente con trastornos relacionados al uso de sustancias psicoactivas, se asoció significativamente con el comportamiento sexual de riesgo, especialmente en los varones. Asimismo, se identificó que la mayor frecuencia de prácticas religiosas y el tener padres casados por lo civil tienen un efecto protector de las prácticas sexuales de riesgo. ${ }^{9}$

Se han realizado pocos estudios cualitativos enfocados a comprender los factores socioculturales asociados a las prácticas sexuales de riesgo en poblaciones psiquiátricas. Collins, Unge y Armbrister ${ }^{10}$ encontraron que la etnicidad en situación de marginación, la condición de inmigrante transnacional, la pobreza y las desigualdades de género favorecen una mayor vulnerabilidad al riesgo de transmisión de VIH en las mujeres hispanas y negras con TMG de Estados Unidos. Sin embargo, no existen reportes relacionados con la construcción de significados de la prevención de las prácticas sexuales de riesgo en las personas que padecen trastorno bipolar.

Este trabajo propone una aproximación al problema de la vulnerabilidad de estas personas, desde el enfoque centrado en la cultura, esto es, concibiendo las voces "subalternas" de los que viven la situación, para que expongan los riesgos y barreras que desfavorecen las acciones preventivas.

Consideramos la cultura como una red dinámica y compleja de significados en flujo constante, que interactúan con los procesos estructurales que la rodean, y que los significados son construidos por los actores. ${ }^{11}$ En este sentido, existe una seria discusión sobre si Internet puede considerarse cultura o más bien un artefacto cultural, lo que replan- tea la distinción entre lo real y lo virtual, y sus implicaciones metodológicas. Por ello se sugiere considerar que la producción del sentido en la interacción cibernética ocurre tomando en cuenta las condiciones en que se emplea la Internet, el contexto en el que se escribe y los espacios sociales que emergen en su uso. ${ }^{12}$

Una comunidad virtual puede ser vista entonces como un espacio donde emergen significados, pero los significados y las percepciones aportados por sus miembros adquieren forma según los entornos de donde provienen. Estudios realizados sobre el impacto social que tiene el uso de comunidades virtuales muestran que esta participación influye en el cambio de opiniones, preferencias y acciones de sus miembros. ${ }^{13}$ Asimismo afecta la toma de decisiones, al implicar un cambio en la percepción sobre lo que acontece, e incluso en sí mismos. ${ }^{14}$ Por lo anterior, podemos suponer que esta interacción social puede inducir cambios en las propias prácticas sexuales y la percepción del riesgo de contagio del VIH.

Este estudio pretende identificar los significados y prácticas sobre la prevención del riesgo de transmisión del VIH en personas que padecen trastorno bipolar y que son miembros activos del weblog "Foro Bipolarmexico".

\section{MATERIAL Y MÉTODOS}

Se realizó un estudio cualitativo bajo el enfoque del método etnográfico de weblogs. ${ }^{12,15}$ Se aplicaron los supuestos de este método para realizar una aproximación holística al entorno de interacción virtual y al proceso de conectividad cultural del weblog "Foro Bipolarmexico", que alberga a una comunidad de personas con TAB.

El muestreo de foros de expresión publicados de septiembre de 2007 -fecha de inicio de la comunidad virtual-a marzo de 2010 fue propositivo. ${ }^{16}$ Se revisó el "índice de foros" y se seleccionaron tres foros que permitieron generar diálogos sobre sentimientos y experiencias respecto a las prácticas sexuales y el riesgo de VIH/SIDA: "Foro general", "Información general" y "Pregúntales a los demás".

Posteriormente, se revisaron los temas que se incluían en cada foro y se eligieron los subtemas que fueron más leídos y/o respondidos por los miembros o visitantes. Esta información se obtuvo del mismo foro, ya que en él se va registrando cada entrada.

Para la organización del material se copiaron las conversaciones de los temas elegidos en un procesador de textos donde se incluyó el enlace que permitió conectarse con la información completa y su contexto discursivo.

Para el análisis de la información, se utilizaron procedimientos de análisis de contenido temático inductivo. ${ }^{17} \mathrm{La}$ codificación de la información implicó la lectura de los textos y su fragmentación en temáticas que luego fueron agrupadas en tres categorías mayores: aspectos psicobiológicos, microsociales y macrosociales. 
Al final, se construyó una síntesis interpretativa basada en las relaciones de los temas de las tres categorías mayores, para generar un modelo sobre la cultura de riesgo y prevención que expresan los miembros del foro.

Para tener acceso al foro y hacer la descripción, la autora principal del estudio se registró en el weblog. Participó primero como visitante sin especificar su condición; posteriormente como familiar de una persona con $\mathrm{TAB}$, y durante el proceso de análisis se presentó ante la audiencia del foro como investigadora y solicitó por escrito al administrador su consentimiento para utilizar la información del weblog. El administrador del foro, así como todos los miembros que contestaron a la publicación, respondieron de forma afirmativa a la petición.

\section{RESULTADOS}

El procedimiento metodológico permitió identificar que el tema de la sexualidad es un campo de interés para personas con $\mathrm{TAB}$, especialmente por las dificultades a las cuales se enfrentan por vivir con este padecimiento. De 592 subtemas incluidos en el primer tema general "Preséntate y cuéntanos tu caso", el "Incremento del deseo sexual" ocupó el lugar 22, con 177 lecturas y 12 respuestas hasta el 8 marzo del 2010.

Este procedimiento metodológico permite apreciar los campos de oportunidad para la investigación en este padecimiento, pues constituyen las necesidades más apremiantes para los miembros de este blog. Entre éstas se pueden resaltar: a) el estigma social de la enfermedad; b) la dificultad para el control de la enfermedad debida a tratamientos poco eficaces, el diagnóstico erróneo y la relación conflictiva con los servidores de la salud mental y c) la falta de información veraz sobre el padecimiento a la sociedad.

A continuación se describen las características de la comunidad virtual y los principales hallazgos sobre los significados de prevención y riesgo, en el marco de las condiciones generadas por el padecimiento.

\section{Características del weblog analizado}

El weblog "Foro Bipolarmexico" (http://bipolarmexico.foros.ws/) fue publicado en Internet en septiembre de 2007. Desde entonces recopila cronológicamente los textos escritos por los miembros de la comunidad virtual -la mayoría mexicanos-, quienes de forma libre comparten sus experiencias.

El creador del sitio, cuyo alias es "Leónidas", padece $\mathrm{TAB}, \mathrm{y}$ construyó la comunidad con un enfoque humanista de apoyo mutuo. Este enfoque se refleja en las narraciones donde varios miembros expresan el apoyo invaluable que brinda este weblog para ellos y sus familias.

El servicio del blog es ofrecido por "foros.ws", un sitio que de forma gratuita proporciona la posibilidad de formar un foro, cuya intención es la de proporcionar una herramienta para crear comunidades. La página del foro contiene las siguientes secciones: a) Inicio, donde se da la bienvenida y se exponen los nuevos materiales; b) Índice de foros; c) Mis favoritos, donde se añaden los temas de mayor interés y d) Rangos, que incluye las listas de los miembros más activos y de las personas inscritas en el blog (710 hasta el 8 de marzo de 2010), así como los servicios de mensajería privada y de grupos de usuarios.

El blog es un espacio donde personas que tienen el padecimiento y sus familiares conforman una comunidad virtual basada en la expresión de comentarios, difusión de fotografías y videos, y desarrollo de diálogos y debates sobre distintos temas.

La participación de los miembros del foro es anónima, lo cual favorece distintas posibilidades de expresión y mecanismos de apoyo social en la interacción. Sin embargo, la forma de participación también implica la posibilidad de que existan miembros denominados "fake" (usuarios que se hacen pasar por otros usuarios o que no tienen el padecimiento ni son familiares de alguien con TAB). Por esto, es importante considerar que podría haber contenidos en el foro que no necesariamente fueron respondidos por la población de interés.

\section{Expresión de significados sobre la prevención}

El análisis de contenido temático permitió identificar tres niveles de expresión de elementos relacionados con la construcción de significados de prevención del riesgo de transmisión del VIH en los miembros participantes del "Foro Bipolarmexico": 1. el nivel de elementos psicobiológicos, 2. el nivel microsocial y 3. el nivel macrosocial. A continuación se describe cada uno de ellos.

\section{Elementos psicobiológicos}

En este nivel se incluyen características inherentes a la enfermedad (episodios maniacos e hipomaniacos, depresivos y el uso de medicamentos) que se identifican como relacionados a prácticas de riesgo y significados de prevención del VIH. En el cuadro 1 se aprecian algunos ejemplos sobre las conversaciones de blogueros que se refieren a cada categoría.

Entre la manía impulsiva y la libido de subsuelo. Hombres y mujeres participantes del foro manifiestan que el TAB por sí mismo y por el uso de medicamentos para controlarlo tienen efectos en la satisfacción sexual y en el autocuidado del cuerpo.

Por una parte, señalan en sus conversaciones que en el estado de manía se experimenta un aumento del deseo sexual y la pérdida del control de impulsos, lo cual lleva en algunos casos a realizar prácticas sexuales de riesgo (ver subcategoría: manía impulsiva). 
Cuadro 1. Elementos psicobiológicos

Categoría: Entre la manía impulsiva y la libido de subsuelo

Sub-categorías Conversaciones de participantes

Manía impulsiva "Mujer (M): [...]' he sentido que últimamente como lo mencioné al principio me dan muchas ganas de tener 'contacto físico' con personas que me gustan, y bueno creo que es normal tener ese deseo por una persona, pero en dos semanas he estado con 3 personas diferentes y cada vez sentía cómo los deseos aumentaban más y más.

Hombre $(\mathrm{H})$ : No te sientas diferente o rara, yo creo que es algo que nos ocurre a la mayoría de los bipolares.... Perdemos el control en el plano sexual, ya sea a la baja o a la alza... Dependiendo del estado de ánimo.... Por lo general el deseo sexual incontrolable se asocia a la manía.

M: [...] es muy posible ke ${ }^{\mathrm{iii}}$ cuando uno está en estado de manía sea uno infiel, por el estado eufórico y el libido elevado, pero hay kienes no lo hacen se controlan en mi caso me cuesta trabajo la verdad jajajaiv pero también no podemos escudarnos en nuestra enfermedad por nuestros actos [...]."

La libido del sub- "M: Pues dile a tu psiquiatra que te ponga [refiere un antidepresivo compuesto por paroxetina] que como efecto secundario suelo: $\quad$ lleva la libido al subsuelo. Y lo digo en serio yo lo tomo y prácticamente no tengo ganas, así no corres riesgo de embarazarte ni de contagiarte de nada."

Disminución "M: [...] tomo [mismo medicamento referido por la otra mujer] también, y déjame decirte que tienes toda la razón, es de la libido y increíble cómo un medicamento puede llegar a hacer eso en mi mente y cuerpo, te lo digo porque empecé a tener pareja medicamentos hace poco, y es IMPOSIBLE prácticamente encontrarme satisfecha sexualmente. La buena noticia es que mi doctora ya me lo quitará en estas semanas (ya tengo la dosis mínima) y pues volveré a ser la misma [...], pero sí, espero seguir controlando eso, aunque prácticamente ya entendí que esos arranques de hipersexualidad me dan más cuando ando en un estado total de manía.

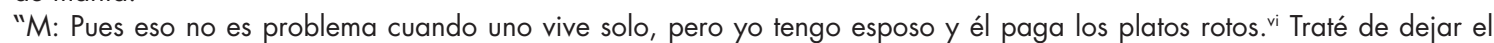
[refiere un antidepresivo compuesto por paroxetina] y el doctor me lo cambió por otra cosa, pero me ponía loquita [...]. Esta vez trataré de hacer un esfuerzo para mantener la normalidad de mi matrimonio, en medio de lo poco que el [refiere un antidepresivo compuesto por paroxetina] me deja hacer."

Disminución de la "M: La depresión es el primer factor de la disfunción sexual + vii él [refiere un antidepresivo compuesto por paroxetina] me libido y depresión incapacita totalmente.

M: Cuando estoy maniaca él está feliz pues se renuevan nuestras relaciones, pero cuando estoy deprimida pasamos semanas sin relaciones sexuales. Increiblemente él se ha adaptado.

$\mathrm{H}:[\ldots]$ en relación con el sexo como dice la compañera, es casi nulo en momentos de depresión, pero no creo que eso le importe a una persona si de verdad le quieres.

$M:[. .$.$] ahorita estoy pasando \mathrm{x}$ una racha difícil en mi matrimonio... Mi libido está baja.... Yo sé que esto no justificaría el proceder de mi esposo... Pero lo entendería y probablemente lo perdonaría.

$\mathrm{H}$ : Desde mi manera de entender el amor, no, no lo perdonaría. Es más, si fuese al revés, que yo sea el infiel rompería con la relación, ya que convivir con eso para toda la vida es como una cicatriz que estará allí para siempre, será motivo de dudas, de malestar, culpa y lógicamente algo, una parte de ese amor, habrá muerto para siempre ....la confianza."

\section{Categoría: Prevención del riesgo: entre la estabilidad fugaz y el descontrol emocional}

\section{Sub-categorías}

Estrategias de prevención

Estabilidad fugaz y descontrol emocional

\section{Conversaciones de participantes}

"M: He hablado con mi psiquiatra de esto y es algo en lo que estamos trabajando ahorita, porque pues sí es preocupante, porque puede ser riesgoso para mí, ustedes saben por la impulsividad... No protección, un embarazo, etc. M: También puedes como dice Pulpo tratar de calmarla con terapia ocupacional, es una buena idea. Yo te recomiendo que si no puedes controlarte hagas lo que quieras pero que te cuides mucho, pues recuerda que no sólo puedes quedar embarazada si no te pueden pasar alguna enfermedad como el VIH.

H: [...] solucionarlo?2?2viii Eso sí está cabrón... @eses un impulso muy fuerte y que hasta parece incontrolable, así que lo único que queda (a mí) es canalizarlo en alguna otra área, ya sea en el arte, la computadora o hasta limpiando mi casa jajaja... Si el impulso ya es mucho más fuerte pues lo satisfago, pero con la mayor precaución posible; pues, como bien dices, se corre el riesgo hasta de -en el remolino del deseo ${ }^{i x}$ - intimar con personas desconocidas que no sabemos qué intenciones puedan tener."

"H: La estabilidad es un lugar donde los bipolares sólo vamos de visita.

M: Que si no será cierto... Yo vivo en la recaída permanente, duro poco en eutimia y zas va de nuevo [...]Y no ha sido ya por dejar de cuidarme, cada vez me cuido más pero parece no dar resultado.

$\mathrm{H}$ : Desde los 21 años que todos los psiquiatras que he visto, me dieron siempre "antidepresivos", de todo tipo, dándome cuenta, recién hace 2 años, que estos "antidepresivos" no son para mi caso. He tomado i.m.a.o., tricíclicos, i.r.s.s., duales, mianserinas, muchos tipos de neurolépticos, "típicos y atípicos ${ }^{x}[. .$.$] pero la cuestión es que, al fin y al cabo, una médica$ psiquiatra que visité hace 2 años ( a los 38 años ) me diagnosticó trastorno bipolar, [...] tengo bastante esperanzas de que esta vez sí, después de 20 años de estar tomando psicofármacos, sea la luz que me esté esperando para la victoria M: [...] con la [refiere un medicamento antipsicótico de segunda generación compuesto por benzisoxazola y piperidina] todo eso engordé muchísimo, me volví una vaca.... Y pa' colmo, me anuló todo deseo sexual... Jajiaja.... Ni loca la vuelvo a tomar.... M: Al final le dije a mi doctor que me negaba a seguir tomando el ácido valpróico y que me buscara otro medicamento. Es que yo me provocaba vómitos, para no engordar y por eso le dije que probáramos con otro. Con el cambio de medicamento, perdí todos los kilos y volvió mi alegría, es que el estar gorda a una la deprime." 
El aumento de deseo sexual es considerado como un problema -principalmente entre las mujeres-, que se puede controlar con el uso de medicamentos, pero que tienen como efecto secundario la disminución del deseo sexual. El medicamento referido por los blogueros es un antidepresivo compuesto de clorhidrato de paroxetina y que es un inhibidor selectivo de la captura de serotonina.

Por otra parte, también perciben que el uso de este medicamento reduce a tal grado su libido, que puede ocasionar por sí mismo un problema importante para quienes tienen vida en pareja. Según perciben algunas mujeres, la disminución de la actividad sexual puede impulsar a sus parejas a tener prácticas sexuales con otras personas.

Un elemento que emerge de las conversaciones es la diferente postura de hombres y mujeres respecto a la "infidelidad" de sus parejas. Para ellas es una circunstancia posible de tolerar cuando disminuyen su actividad sexual, lo que no es así para los varones (ver subcategoría: la libido de subsuelo).

Prevención del riesgo: entre la estabilidad fugaz y el descontrol emocional. Los y las participantes del foro que perciben el riesgo de transmisión del VIH y embarazos no planeados cuando experimentan el aumento del deseo sexual expresaron posibles estrategias para prevenir prácticas riesgosas. Mencionan en sus conversaciones la búsqueda de apoyo en la terapia psiquiátrica, la canalización de los impulsos con terapias ocupacionales y el uso de anticonceptivos y de protección contra enfermedades cuando el impulso sexual es "incontrolable" (ver subcategoría: estrategias de prevención).
Sin embargo, para algunos miembros del foro, las oportunidades de uso de estrategias de prevención se ven disminuidas por la inestabilidad emocional que caracteriza al TAB. Dicha inestabilidad se origina en la falta de control del TAB, la baja efectividad de los distintos tratamientos y el rechazo de los pacientes a utilizar medicamentos por sus efectos colaterales. Respecto a la baja efectividad de los tratamientos, un miembro del foro señaló que, en su caso, la prescripción médica fue incorrecta debido al diagnóstico erróneo que hicieron de su padecimiento por años.

En lo que respecta a los efectos colaterales de los tratamientos farmacológicos, la disminución del apetito sexual y el aumento de peso generó un gran rechazo hacia los medicamentos entre los participantes del foro (ver subcategoría: estabilidad fugaz y descontrol emocional).

De ahí que las estrategias de prevención que ellos sugieren se deban considerar en el marco del control del TAB.

\section{Nivel microsocial del riesgo}

Este nivel abarca interacciones sociales relacionadas con la percepción del padecimiento y su impacto en sus relaciones de pareja. En el cuadro 2 se aprecian algunos ejemplos sobre las conversaciones de blogueros que se refieren a la categoría.

Efecto de la percepción social negativa. El control del TAB y sus efectos en las prácticas sexuales no sólo tienen que ver con cuestiones médicas sino también con la percepción negativa del padecimiento.

Cuadro 2. Nivel microsocial del riesgo

\section{Categoría: Efecto de la percepción social negativa}

\begin{aligned} \hline Sub-categorías & Conversaciones de participantes \\ \hline Aislamiento social & "M: [...] Lo que sí me afecta es la carencia de amigos a veces me pregunto jqué pasa conmigo? Qué tengo? Soy muy \\ & sangrona o quéę Jajajaj.[...]. Casi no salgo y tengo años sin tener novio y ni siquiera he besado a nadie en 5 años jejej. \\ & H: Nunca he tenido novia, a una muchacha que amé con locura me dio vergüenza decirle que era bipolar y la dejé de \\ & ver, gran error que me dolerá toda la vida porque ya se casó... Nunca he tenido un beso de amor con alguien, sí me han \\ & besado, pero cuando he pagado por sexo. Así de lastimosa es mi situación en lo que respecta a relaciones sentimentales. \end{aligned}
Locura y discrimi- No me apena decirlo, no me apena decir la verdad."

nación $\quad$ "H: [...] un brother ${ }^{\times i}$ que no sabe de mí me contó que conoció a una chava no le gustaba mucho pero se arreglaba un buen y le entraba a la fiesta cañón, hasta ofreció conectármela después, porque llevaban un mes y ya se habían acostado como 10 veces, el punto es que ella al creer que este wey quería algo serio, le contó que era bipolar, resultado: la fue dejando poco a poco, y ante nosotros ya le decía la "lore" (lorenzaxii), se acostó con ella hasta cansarse y adiós! Al llamarle la chava (como es normal estés enfermo o no) para pedirle explicaciones, él ya sentía que lo acosaba y lo asfixiaba que lo iba a estar siguiendo y alucines así, al preguntarle porq pensaba eso, me dijo q ella estaba loca, que él ya había visto en Parejas estables la T.V. lo que hacían los bipolares!!!"

y género $\quad$ "M: [...] tengo 4 años de casada mi esposo es muy buena persona [...] cuando nos casamos no le dije de mi enfermedad porque yo misma me negaba a creerlo [...] el problema es que yo sigo en las mismas y él ha empezado a cambiar, se sale solo. Hablé con él sobre separarnos, no quiero seguir arruinándole la vida, yo sé que vivir con una persona como yo es muy difícil, pero él no quiere dejarme y yo la verdad no mejoro nada, no sé qué hacer.

M: [...], mi vida familiar y de pareja se ha deteriorado mucho. Mi marido me apoya muchísimo pero por supuesto, se cansa y harta. Lo entiendo y le agradezco todo-todo-todo. Me ha repetido lo cansado q es vivir conmigo. ¡Hasta cuándo va a aguantar, hasta cuándo va a durar su paciencia y amor? Está intentando amar al "nuevo ser" que soy, pero me da pavor el pensar q estoy como a prueba, con ojos inquisidores ante mis actos.

H: Yo amo muchísimo a mi esposa y, sin embargo muchas veces le he hecho daño (sólo moral, nunca físico) [...] cuando estoy consciente me doy cuenta de que la he herido... Esto por supuesto que de ordinario va minando el amor... [...] la enfermedad la vivimos los que la padecemos y los que están a nuestro lado..." 
Cuadro 3. Nivel macrosocial del riesgo

\section{Categoría: Estigmatización social: la satanización está de moda}

Sub-categorías Conversaciones de participantes

Estigma y culpa "H: Les cuento que hace dos años mi mundo se puso de cabeza. En el trabajo comencé a distorsionar la realidad al punto de que pensé que había una conspiración en mi contra... Jeje, [...] Bien, pues yo tenía un par de amigos en aquel entonces. Eran de esos amigos "inseparables" [...]. Ellos fueron testigos de cómo gradualmente fui evolucionando a ese estado y fue precisamente en esa medida que se fueron paulatinamente tratando de desvincular de mí. [...]Pasó un año y ahora estaba deprimido. Mi psiquiatra me recomendó contactarlos en función de arreglar pendientes, de combatir mi aislamiento y con fines de la motivación que necesitaba en ese momento. Así lo hice.[...] Sin embargo no me buscaban, [...] Pensé: Está bien, una cosa es que se alejen cuando estoy teniendo un cuadro paranoide, pero otra cosa muy distinta es rechazarme en este momento que no hay manía [...] Mi psicóloga insiste mucho en que piense en lo que ellos sintieron cuando mi "locura" [...] me dice que reconozca que yo todavía estoy lejos de estar bien, [...] que eso contribuye al rechazo. Y a mí, no sé, se me hace como poner muchas excusas para justificar sus acciones [...] También me dice que me dé cuenta que la cordura es un valor altamente preciado socialmente y que por lo tanto la falta de ella es altamente estigmatizada. [...] ¿ंPero de qué se trata? ¿̇e aprobar todo lo que es entendible, inclusive las acciones que tienen origen en la estigmatización?"

Moda bipolar $\quad \mathrm{H}$ : Por supuesto. Hay una enorme estigmatización y coincido que mucha de la falta de información proviene de la televiy satanización sión, fuente de otros tantos males.

M: últimamente me tiene muy molesta algo, por todos lados la palabra bipolar suena y eso no es lo que me molesta, sino el uso que le dan a ésta $(3$, ahora resulta que todo aquel que se enoja grita o simplemente tiene mal carácter es bipolar. En la tele he visto infinidad de reportajes y pienso que está bien que la gente sepa más sobre lo que es la bipolaridad pero ¿No tiene la impresión de que de alguna forma nos satanizan......?

$M$ : [...] es verdad sí somos capaces y dignos de vivir una vida linda, y tranquila y feliz, sin estigmas horrorosos que devalúen nuestra integridad.

$\mathrm{H}:[\ldots]$ series como CSI, brother's and sister's, twenty-four hours, y ahora en México, mujeres asesinas, como son programas policíacos casi siempre terminan poniendo al final "tal fue diagnosticado como bipolar con colopatía esquizofrénica" o sea!!!, "ojo" el término es explícito, lo cual nos guste o no, en los que siguen estas series (que son millones) va generando la asociación de bipolar=asesino, ¿̇ahora me entienden lo grave de esto? [...] después, ayer en la noche salí con una chava a cenar, en la sobremesa le pregunte que qué le gustaba, entre otras cosas me dijo que las series y me empezó a contar de algunas de las q les mencioné y al contarme un capítulo salió lo de los bipolares. En su cabeza ya tiene bien clavado que el bipolar sólo causa problemas (jaja y sí, pero no siempre), pero o sea ella nunca andaría con un bipolar porq son locos alucinados asesinos, porq piensa eso? Obvio gracias a la T.V.!!! Bueno ahí se los dejo para reflexión! Y no se preocupen bros' ahora yo me acostaré con ella hasta cansarme para vengar a la chava que mi cuate trató mal!Jajajaja no es cierto, seguro no la vuelvo a ver por tapada!!"

Información $\quad$ "H:[...]El padecimiento puede ser enfrentable hay algo que me molesta y es la apertura de los medios de comunicación, certera de la donde se nos hace ver como bichos raros"

bipolaridad "M: además de la estigmatización existente, nosotros mismos nos boicoteamos al no investigar sobre nuestra enfermedad y hacer que el resto de la gente se entere de las características de la misma; la ignorancia se combate con información y es a nosotros a quienes toca hacer que esa información llegue a más y más gente..."

\section{Categoría: El potencial de la genialidad y la sensibilidad}

Conversaciones de participantes

"M: Saben yo veo a cada bipolar como un genio, tenemos otra perspectiva de ver la vida, y una sensibilidad enorme $\mathrm{H}:[. .$.$] podemos hacer cosas portentosas, tenemos esa capacidad. Bipolares y sanos, no hay que discriminar.$

M: A través de todas estas personas es evidente que el trastorno bipolar puede ser manejado. Las personas que sufren trastorno bipolar realmente tienen mucho que ofrecer a este mundo.

$\mathrm{H}$ : [...] comparáramos al trastorno bipolar con alguna cosa. Yo lo comparé con una motosierra. Pues cuando a mí me dijeron que tenía la condición, tomé la motosierra y como no sabía usarla, destruí muchas cosas a mi alrededor, me hice muchas heridas sin sentido y muy dolorosas, porque no había querido aceptarlo como algo potencialmente constructivo. Este trastorno es como una motosierra, que si investigas como está formada, y como usarla correctamente, puedes usarla para hacer esculturas maravillosas con los materiales más duros, aunque cueste dominarla..."

i Aunque se trató de integrar el texto de las conversaciones, tal y como aparece en el blog; algunos párrafos fueron editados, eliminando fragmentos poco relevantes para la discusión que mantenían los blogueros, estas ediciones se aprecian en los espacios en que aparecen los corchetes [...]. Además se modificaron un poco los textos para que fuesen más comprensibles, únicamente agregando acentuaciones en palabras y puntuación en algunas oraciones.

ii Los puntos suspensivos que no están entre corchetes, son parte del texto que escriben los mismos blogueros; no refieren a la edición a la que se hace mención en la nota anterior.

ii Los blogueros tienden a modificar las palabras utilizando letras que no corresponden a la palabra reconocida oficialmente, por ejemplo la expresión "Ke", en lugar de "que", o bien escriben palabras incompletas como "porq" para decir "porque" $y$ " $q$ " para "que", sin embargo se comprenden y reconocen entre los usuarios.

"La paroxetina es un antidepresivo, inhibidor selectivo de la recaptura de serotonina. Utilizado principalmente para el tratamiento de la depresión de diversos tipos, incluyendo la depresión reactiva y grave, y la depresión acompañada por ansiedad.

reactiva y grave, y la depresión acompañada por ansiedad.
vi Esta expresión "él paga los platos rotos" se utiliza en el contexto para referirse a quien le afecta una situación que es provocada por otro sujeto.

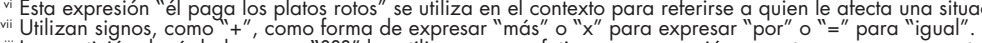

viii La repetición de símbolos como "???"?" los utilizan para enfatizar una expresión, en este caso una pregunta.

ix Algunos blogueros utilizan metáforas para referir sus emociones, experiencias y expectativas cómo "Remolino del deseo" o "la luz que me esté esperando para la victoria", así como analogías para representar de forma divertida sus pensamientos sobre su apariencia social "estar como vaca" "bichos raros".

analogías para representar de forma divertida sus pensamientos sobre su apariencia social "estar como vaca", "bichos raros". “ Es importante resaltar que los blogueros manejan términos especializados

xi así como multiplicidad de nombres de medicamentos que han utilizado.

xii Expresiones como "lore" "Lorenza" es una forma peyorativa que relacionan con la condición de enfermedad mental, en el contexto se puede entender como tonto, falto de cordura, entre otros. 
Existen condiciones sociales que se relacionan con tales prácticas, entre ellas se encuentra el aislamiento social que desfavorece la formación de parejas estables por temor a sufrir rechazo. Una mujer del foro destaca su situación de aislamiento, y un hombre habla de la opción de acudir al sexo comercial ante la dificultad de establecer relaciones sentimentales firmes (ver subcategoría: aislamiento social).

Cuando las personas con trastorno bipolar tienen una vida sexual activa y sus parejas perciben la enfermedad como una locura, estas últimas no toman la relación en serio y tienden a abandonarlas. Es lo que describe un participante del foro quien comenta que un amigo se ofreció a presentarle a su pareja bipolar (ver subcategoría: locura y discriminación).

Por otra parte, la dinámica de las parejas maritales basada en una percepción negativa de la enfermedad genera conflictos sentimentales expresados en rechazo y culpabilidad. En el caso de las mujeres, el sentimiento de culpabilidad y el autopercibirse como indignas del amor y apoyo de sus parejas llegan a justificar la violencia psicológica que éstas ejercen. Por su parte, los varones sufren por los enfrentamientos de pareja que surgen cuando se manifiesta el trastorno con agresiones, lo que debilita la relación de pareja (ver subcategoría: parejas estables y género).

\section{Nivel macrosocial del riesgo}

En este nivel se integra la dimensión del estigma social de la enfermedad y su impacto en las relaciones de pareja. En el cuadro 3 se aprecian algunos ejemplos sobre las conversaciones de blogueros que se refieren a cada categoría.

Estigmatización social: la satanización está de moda. El rechazo social a las personas con trastorno bipolar se basa en la incomprensión y la percepción social que los ubican como locos y faltos de cordura. Esta estigmatización es fortalecida al depositarse la responsabilidad en quien tiene el trastorno, en lugar de fomentarse una cultura de información donde se abone a la comprensión de estas personas (ver subcategoría: estigma y culpa).

Algunos miembros del blog critican la situación de estigma, generado por la falsa información sobre el trastorno bipolar que se ha difundido por los medios masivos de comunicación. La alta frecuencia de notas y reportajes sobre el tema ha puesto "de moda" el asunto, sin aclarar la mayoría de las veces en qué consiste este diagnóstico, el que es aplicado indistintamente a quienes tienen conductas "extrañas"; así pues, el tratamiento de la información ha favorecido la satanización de personas con TAB.

Dos mujeres del foro señalan que esta "moda" ha propiciado el rechazo, discriminación y devaluación de su integridad por los pares. Asimismo, "marca" sus relaciones interpersonales, influyendo en sus prácticas sexuales. Al estigmatizarse masivamente el TAB, las personas que rodean a quienes lo padecen rechazan la posibilidad de conformar una relación estable y ellos mismos se observan discriminados, "satanizados", por lo que tienden a conformar relaciones de pareja ocasionales o bien son sujetos de abuso por sus parejas, especialmente en el caso de las mujeres (ver subcategoría: moda bipolar y satanización).

En el foro se ha planteado la necesidad de que en los medios se hable de forma positiva sobre el trastorno y que se difunda información de manera correcta. Uno de los temas propuestos para la discusión entre los miembros se titula: "No somos bonitos pero estamos a la moda". Según lo manifestado, lo más difícil de enfrentar en su padecimiento es el estigma social (ver subcategoría: información certera de la bipolaridad).

El potencial de la genialidad y la sensibilidad. Como se describió previamente, el estigma percibido propicia el aislamiento social en las personas con este padecimiento, lo cual se relaciona con prácticas riesgosas como tener relaciones sexuales con desconocidos o con trabajadores/as del sexo comercial. Asimismo, la autodevaluación que se experimenta por el rechazo social se puede manifestar en el alejamiento de los seres queridos y en el abuso de sus parejas sexuales.

Además de plantear el hacer frente al estigma social mediante la información correcta del padecimiento como estrategia para evitar el aislamiento social y el abuso, los participantes del blog insisten en reconocer sus potencialidades, utilizando de referencia a personajes famosos que padecen el trastorno. Entre éstas destacan tener una perspectiva diferente sobre la vida y una sensibilidad especial.

Un participante del foro expresa la metáfora de la motosierra; él compara esta herramienta con la bipolaridad al mostrar que el desconocimiento sobre su uso y características es riesgoso por los daños que puede provocar a sí mismo y a los demás. Sin embargo, cuando existe una instrucción sobre la misma, es posible controlarla y utilizarla de forma positiva para mostrar su potencial (ver: Potencial de la genialidad y la sensibilidad).

\section{DISCUSIÓN}

Este estudio realizó una etnografía de la comunidad virtual llamada "Foro Bipolarmexico", un espacio de interacción social donde existe identificación y apego entre sus miembros, que brinda acompañamiento en los procesos de angustia y que ofrece información basada en la experiencia.

Los significados de prevención en personas que padecen trastorno bipolar parecen verse condicionados por diversos factores psicobiológicos, microsociales y macro sociales, que a su vez están entrelazados e implicados en las prácticas sexuales de esta población.

La conversación sobre temas de interés y sobre sus vivencias ofreció un recurso valioso para el desarrollo de esta investigación que se interesó en comprender los significados socialmente construidos sobre la prevención de conta- 
gio por el VIH. Los subtítulos: Entre la manía impulsiva y la libido de subsuelo, Prevención del riesgo: entre la estabilidad fugaz y el descontrol emocional, Efecto de la percepción social negativa, Estigmatización social: la satanización está de moda y El potencial de la genialidad y la sensibilidad ilustran algunos significados construidos por los participantes, donde se muestran además las condiciones que pueden estar implicadas en la vulnerabilidad frente al riesgo de contraer el VIH.

Los episodios inherentes a la enfermedad y el medicamento, la poca discusión sobre temas sexuales entre quien vive con el padecimiento, los profesionales de la salud y sus parejas, y el estigma de la enfermedad fueron los tres pilares donde se aprecian las condiciones que vulneran a esta población y las estrategias de prevención que se han sugerido para contrarrestarla.

Las alteraciones en el deseo y la actividad sexual durante los episodios maniaco, hipomaniaco y depresivo concuerdan con la literatura clínica. ${ }^{18}$ Asimismo, la impulsividad y el aumento de la actividad y deseo sexual que experimentan los blogueros en la manía también ha sido registrada en estudios realizados en diferentes países, donde se muestra que la hipersexualidad y la disminución en el control de impulsos se presentan entre el 40 y $60 \%$ de la población durante estos episodios. ${ }^{19}$ Por su parte, otros estudios muestran asociaciones entre las prácticas de riesgo en el episodio maniaco reciente y la comorbilidad del uso de sustancias psicoactivas. ${ }^{8,9}$

En el caso del episodio depresivo, identificamos que su sintomatología y el uso de medicamentos disminuyen el deseo y la actividad sexual, evidencia que es similar a trabajos clínicos donde se relaciona el episodio depresivo y algunos tratamientos farmacológicos como antidepresivos, antipsicóticos atípicos, así como los estabilizadores del estado de ánimo (como el litio y antiepilépticos), con la actividad o la satisfacción sexual de la persona. ${ }^{20}$ Dicha disminución del deseo y actividad sexuales en los episodios depresivos podría pensarse que lleva a una disminución de comportamiento riesgoso para la transmisión de VIH, sin embargo esto no siempre ocurre. En adolescentes con depresión se han identificado asociaciones entre la sintomatología y prácticas sexuales riesgosas para la transmisión del VIH. ${ }^{21}$

En el presente estudio encontramos que en la disminución de la actividad sexual hay nula percepción del riesgo; sin embargo, algunas mujeres expresan que esta situación lleva a sus parejas a buscar relaciones ocasionales. Esta dinámica de pareja, donde la mujer conoce y acepta en cierta medida las prácticas sexuales de su pareja, es poco visibilizada como una dinámica que las vulnera frente al riesgo de transmisión del virus.

Por su parte, el efecto de la disminución del deseo y la actividad sexual que los participantes han experimentado con algunos medicamentos lleva a pensar que algunos de ellos podrían utilizarse para contrarrestar la hipersexualidad en la manía. Sin embargo, la autoadministración de me- dicamentos antidepresivos cuyo compuesto es la paroxetina podría agravar su padecimiento, considerando que el uso no vigilado de estos medicamentos y en ausencia de un estabilizador del estado de ánimo puede resultar en un viraje a la manía. ${ }^{22}$

Esta investigación muestra una preocupación importante de los miembros del blog ante el aumento de su deseo sexual y sus prácticas sexuales en los episodios maniacos, y por la disminución de la actividad sexual en la depresión y por el uso de medicamentos. Asimismo, destaca una carencia de conocimientos, principalmente en los que tienen poco tiempo con el padecimiento, sobre el trastorno y el efecto de los medicamentos.

Como medidas de prevención en este nivel, que se puede denominar psicobiológico, los participantes sugieren que el impulso sexual incontrolable que a veces experimentan sea canalizado a actividades recreativas, o se solicite un cambio de medicamentos o bien, si esto no funciona, se utilicen preservativos.

Esto supondría que algunas personas con trastorno bipolar podrían presentar sintomatología relacionada con prácticas sexuales de riesgo y tendrían que ser atendidas de forma individual. Sin embargo la sintomatología no es determinante del riesgo, existen otros factores psicosociales y estructurales involucrados en la vulnerabilidad y que a su vez se deben considerar como pilares en la prevención: a) informar sobre la sintomatología y su efecto en el deseo y función sexual a quien tiene la enfermedad y a sus familiares para que exista una detección oportuna de alteraciones relacionadas a la sexualidad, y se puedan generar estrategias psicoeducativas en salud sexual y/o farmacológicas; $b$ ) fomentar la discusión abierta del tema de la sexualidad, medidas de prevención y riesgo de contagio con el VIH con sus relaciones de pareja y c) identificar si existe una percepción estigmatizada del riesgo en sus vidas, así como la forma en que esta percepción se ha construido socialmente como personas con un padecimiento mental.

El desconocimiento sobre el efecto que tienen el trastorno bipolar y los medicamentos en las prácticas sexuales provoca angustia e incomprensión en quienes viven con este padecimiento y en sus parejas. Un estudio realizado en las parejas de personas con TAB encontró que los conflictos se generan principalmente durante los episodios maniacos y cuando hay un desconocimiento de dichas alteraciones. ${ }^{23}$

Los efectos de la desinformación sobre la sexualidad son de tal magnitud que no sólo generan conflictos conyugales y un riesgo para la transmisión del VIH. Un trabajo realizado en personas con $\mathrm{TAB}$ encontró que las alteraciones en su vida sexual se asociaron de forma significativa con los intentos suicidas. ${ }^{4}$

Los y las participantes de la comunidad virtual buscaron respuestas dentro del foro frente a sus necesidades y a la ausencia de información que debiera ser proporcionada por los profesionales de la salud mental. Existe evidencia empí- 
rica de que éstos discuten poco o nunca aspectos tales como las posibles alteraciones en la sexualidad y sobre la prevención del VIH/SIDA con pacientes que padecen TMS; ${ }^{24}$ la mayoría de los participantes en investigaciones al respecto señalan que obtuvieron más información de otras fuentes. ${ }^{25,26}$ Aun cuando se ha señalado en numerosos estudios que es necesario que los psiquiatras y demás profesionales de salud mental brinden información y capacitación sobre las ITS, el VIH/SIDA y las prácticas sexuales riesgosas, poco se ha hecho al respecto. $23,27-29$

En este sentido, una percepción negativa de la enfermedad o el desconocimiento sobre la sintomatología en el aspecto sexual puede generar conflictos de pareja y abandono. Los mismos blogueros proponen que estos temas se deben discutir con sus psiquiatras y con sus parejas a fin de lograr una mayor comprensión y cuidado de su cuerpo.

En gran parte, la falta de información se debe al estigma de la enfermedad mental; las personas con trastorno bipolar, como la mayoría de personas con trastornos mentales graves, son invisibles como un grupo vulnerable con necesidades de atención, con capacidades de producción y desarrollo social; y sólo son visibles para la discriminación.

El estigma es un elemento estructural en la comprensión de los significados sobre la prevención y de sus prácticas sexuales. Los miembros de la comunidad virtual exponen y critican la estigmatización que viven, expresada en el rechazo, discriminación y devaluación de su integridad como seres humanos. Algunos estudios han abordado el tema del estigma en personas con $\mathrm{TAB}$ y han encontrado que sus relaciones interpersonales se ven minadas, así como su posibilidad de control de la sintomatología, lo que genera un mayor deterioro. ${ }^{30,31}$

Los blogueros discuten de forma extensa esta barrera social, tanto como una condición que promueve la desinformación como un fenómeno que influye en el tipo de relaciones sociales y de pareja que emprenden. Para algunos de ellos, las relaciones sexuales con parejas ocasionales o con trabajadoras del sexo comercial forman parte de la cotidianeidad debido a la poca estabilidad que tienen en sus relaciones de pareja, lo que les hace sentirse enfermos, despreciados y devaluados por sus síntomas.

Estos resultados coinciden con dos estudios realizados en los Estados Unidos y en el Brasil, pues muestran la influencia del estigma en el tipo de relaciones que tienen las personas con TMS, con la imposibilidad de la discusión directa de su sexualidad y la escasa prevención de las ITS. ${ }^{32,33}$ En este caso, los blogueros promueven que se les reconozcan socialmente sus capacidades y se les informe sobre el trastorno de forma adecuada.

Algunos recursos identificados para la prevención del riesgo a nivel microsocial y macrosocial son: la discusión abierta sobre el efecto del padecimiento y medicamentos en las prácticas sexuales tanto con los pacientes como con los familiares; promover la información adecuada sobre el padecimiento por los medios masivos de comunicación; y fomentar el apego a redes de apoyo social como esta comunidad virtual.

Finalmente, la dimension de género emerge entre los datos como una línea para desarrollar por las implicaciones diferenciales que aparecen entre hombres y mujeres en sus discusiones. Las mujeres con el trastorno son más vulnerables al riesgo por abuso y coacción sexual que los varones. Por su parte, los hombres son mayormente vulnerables al riesgo de aislamiento social asociado al estigma, que se expresa en una menor frecuencia de relaciones de pareja estables y relaciones con múltiples parejas ocasionales y a su vez en una menor discusión sobre la actividad sexual de sus parejas. Esta evidencia se relaciona con estudios realizados en países anglosajones con población que padece TMS, que señalan que existe un riesgo significativamente mayor en los hombres de infectarse de VIH, por la multiplicidad de parejas sexuales, ${ }^{7}$ aunque también muestran más estrategias de prevención, como el uso más frecuente del condón que las mujeres. ${ }^{34}$ Además las mujeres con trastornos mentales graves muestran prevalencias elevadas de abuso sexual. ${ }^{35,36} \mathrm{Sin}$ embargo, se requiere de un trabajo más especializado que abone al análisis de los discursos generados por los hombres y por las mujeres para llegar a una comprensión más profunda de las condiciones socioculturales inmersas en la experiencia del padecimiento y en sus prácticas sexuales, incluida una perspectiva de género.

\section{LIMITACIONES DEL ESTUDIO}

La evidencia aquí generada se debe considerar como parte de una población que compone esta comunidad virtual, que tiene acceso a Internet, a los recursos materiales y a los educativos básicos para conversar en este blog. También es una población que tiene la posibilidad económica de ser atendida por los servicios de salud mental formales; por ejemplo: la psiquiatría y la psicoterapia. Es necesario abordar aquella población que carece de estos recursos, que se encuentra en situación marginal y que posiblemente muestre diferentes condiciones de riesgo e incluso mayor vulnerabilidad. Recordemos que la pobreza es un predictor para el mayor número de prácticas sexuales de riesgo, ${ }^{35}$ según estudios realizados en adultos estadounidenses y mujeres puertorriqueñas con TMS que habitaban en Estados Unidos. La situación de pobreza ha sido poco investigada y aún menos se ha comparado a personas que padecen trastornos mentales graves de diferentes estratos socioeconómicos.

\section{AGRADECIMIENTOS}

Los autores agradecen de manera especial a "Leónidas", administrador de la comunidad virtual "Foro bipolarmexico", y a todos los miembros por su consentimiento para utilizar la información registrada en el foro, la cual constituye el centro de este trabajo. 


\section{REFERENCIAS}

1. Joska J, Kaliski S, Benart S. Patients with severe mental illness: A new approach to testing for HIV. S Afr Med J 2008;98(3):213-217.

2. Raja M, Azzoni A. Sexual behavior and sexual problems among patients with severe chronic psychoses. Eur J psychiatry 2003;18(2):42-48.

3. Sacks M, Dermatis H. Acute psychiatric illnes: effects on HIV-risk behavior. Psycho Soc Rehabil J 1994;17(4):1.

4. Dell'Osso L, Carmassi C, Carlini M, Rucci P et al. Sexual disfunctions and suicidality in patients with bipolar disorder and unipolar depression. J Sexual Medicine 2009;6(11):3063-3070.

5. Carey M, Carey K, Maisoto S, Schroder K et al. HIV risk behavior among psychiatric outpatients: Association with psychiatric disorder, substance use disorder, and gender. J Nerv Ment Dis 2004;192(2):289-296.

6. Volavka J, Convit A, Czbor P, Douyon R et al. HIV Seroprevalence and risk behaviors in psychiatric inpatients. Psychiatry Res 1991;39:109-114.

7. Sacks M, Silberstein C, Weiler P, Perry S. HIV-related risk factors in acute psychiatric inpatients. Hosp Community Psychiatr 1990;41(4):449-451.

8. Meade $C$, Graff F, Griffin M, Weiss R. HIV risk behavior among patients with co-occurring bipolar and substance use disorders: Associations with mania and drug abuse. Drug Alcohol Dependence 2008;92(1-3):296-300.

9. Bakare M, Agomoh A, Ebigbo P, Onyeama G et al. Co-morbid disorders and sexual risk behavior in Nigerian adolescents with bipolar disorder. Int Arch Med 2009;2:1-5.

10. Collins $P$, Unger $H$, Armbrister A. Church ladies, god girls, and locas: Stigma and the intersection of gender, ethnicity, mental illness, and sexuality in relation to HIV risk. Soc Sci Med 2008;67:389-397.

11. Dutta M. Communicating about culture and health: Theorizing culture-centered and cultural sensitivity approaches. Commun Theory 2007;17:304-328.

12. Hine C. Etnografía virtual. Barcelona: UOC; 2004.

13. Bagozzi R, Dholakia U. Intentional social action in virtual communities. J Interactive Marketing 2002;16(2):2-21.

14. Dholakia U, Bagozzi R, Pearo L. A social influence model of consumer participation in network and small group-based virtual communities. Int J Res Mark 2004;21:241-263.

15. Ferrada M. Etnografía, un enfoque para la investigación de Weblogs en Biblioteconomía y documentación. Biblios 2006;7(23).

16. Patton M. Qualitative evaluation and research methods. Londres: Sage; 1990.

17. Mayring P. Qualitative content analysis. Forum Qual Soc Res 2000;1(2).

18. American Psychiatric Association. DSM-IV-TR: Manual de diagnostico y estadistico de los trastornos mentales. Barcelona: Masson; 2002.

19. González-Pinto A, Aldama A, Sonsoles A, González R. Sexualidad en el trastorno bipolar. En: Montejo. Sexualidad y salud mental. Barcelona: Glosa; 2005.

20. Montejo A, Grupo español de trabajo para el estudio de disfunciones sexuales. Disfunción sexual provocada por antipsicóticos. Impacto clí- nico y propuestas de actuación. En: Montejo. Sexualidad y salud mental. Barcelona: Glosa; 2005.

21. Sherier L, Harris S, Sternberg M, Beardslee W. Associations of depression, self-esteem, and substance use with sexual risk among adolescents. Prev Med 2001;33(3):179-189.

22. Reiser R, Thompson L. Trastorno bipolar, avances en psicoterapia, práctica basada en la evidencia. México: El Manual Modero; 2006.

23. Lam D, Donaldson C, Brown Y, Malliaris Y. Burden and marital and sexual satisfaction in the partners of bipolar patients. Bipolar disord 2005;7(5):431-440.

24. Rosenberg K, Bleiberg K, Koscis J, Gross C. Asurvey of sexual side effects among severely mentally ill patients taking psychotropic medications: impact on compliance. J Sex Marital Ther 2003;29(4):289-296.

25. Coverdale J, Arukk J. AIDS and family planning counseling of psychiatrically ill women in community mental health clinics. Community Ment Health J 1992;28(1):13-20.

26. Coverdale J, Schotte D, Ruiz P, Pharies $S$ et al. Family planning needs of male chronic mental patients in the general hospital psychiatry clinic. Gen Hosp Psychi 1994;16(1):38-41.

27. Harírí A, Karadag F, Gökalp P, Aksoy U. Awarness of sexually-transmitted diseases and risky sexual behavior in bipolar patients: A comparative study with healhy controls. Nöropsikiyatri Arsivi 2009;46(2):52-60.

28. Harírí G, Karadag F, Gökalp P, Essizoglu A. Risky sexual behavior among patients in Turkey with bipolar disorder. J Sex Med 2011;8(8):2284-2291.

29. McCandless F, Sladen C. Sexual halth and women with bipolar disorder. J Adv Nurs 2003;44(2):42-48.

30. Perlick D, Rosenheck R, Clarkin J, Sirey J et al. Stigma as a barrier to recovery: Adverse effects of perceived stigma on social adaptation of persons diagnosed with bipolar affective disorder. Psychiatr Serv 2001;52:1627-1632.

31. Vázquez G, Kapczinskj F, Magalhaes P, Córdoba R et al. Stigma and functioning in patients with bipolar disorder. J Affect Disord 2011;130(1-2):323-327.

32. Collins $\mathrm{P}$, Unger $\mathrm{H}$, Sweetland A, Wright $\mathrm{E}$ et al. Relationship of stigma to HIV risk among women with mental illness. Am J Orthopsychiatry 2008;78(4):498-506.

33. Elkington $K$, Mckinnon $K$, Gruber $C$, Collins P et al. Percived mental illness stigma and HIV risk behaviors among adult psychiatric outpatients in Rio de Janeiro, Brazil. Community Ment Health J 2010;46(1):56-64.

34. Knox M, Boaz T, Friedrich M, Dow M. HIV risk factors for persons with serious mental illness. Community Ment Health J 1994;30(6):551-563.

35. Goldman E, Loue S, Sajatovic M, Tisch D. Impact of psychiatric and social characteristics on HIV sexual risk behavior in puerto rican women with severe mental illness. Soc Psychiat Epidemiol 2010;45(11):1043-1054.

36. Goodman L, Fallot R.HIV risk behavior in poor urban women with serious mental disorders. Am J Orthopsychiatry 1998;68(1):73-83.

Artículo sin conflicto de intereses 